\title{
Remedial interventions for developmental dyslexia: how neuropsychological evidence can inspire and support a rehabilitation training
}

\author{
Alice Cancer - Alessandro Antonietti \\ Department of Psychology, Catholic University of the Sacred Heart, Milan, Italy \\ DOI: http://dx.doi.org/10.7358/neur-2017-022-canc \\ alice.cancer@unicatt.it
}

\begin{abstract}
Developmental dyslexia (DD) is associated with deficiencies in temporal processing of auditory stimuli, depending on atypical oscillatory neural activity, that are considered to contribute to phonological and reading impairments. To induce a more accurate entrainment to the spectral properties of auditory stimuli in dyslexic readers, we explored the possibility to synchronize speech prosody during reading with an acoustic stimulus presented at a regular pace. Accordingly, an intervention program for $D D$, called Rhythmic Reading Training, was devised. Several test-training-retest studies supported the efficacy of this new methodology on reading skills of primary and highschool students with DD under different conditions. Finally, preliminary results of the application of RRT combined with neuromodulation (i.e., tDCS) in undergraduate students with $D D$ are presented.
\end{abstract}

Keywords: Reading; Developmental dyslexia; Rhythm; Intervention; Neuromodulation; tDCS 


\section{INTRODUCTION}

Developmental dyslexia (DD) is a specific learning disability with a neurobiological origin characterized by an impairment in the ability of reading, that is, not the consequence of intellectual deficit, sensory dysfunction, socioeconomic disadvantage, or lack of educational opportunities (e.g., Snowling \& Hulme, 2012). Behavioural manifestations of DD, along with the estimated prevalence in school-population, vary across languages, due to differences in orthographic depth (i.e., the consistency and transparency of grapheme-phoneme correspondences in written language) (Richlan, 2014; Snowling \& Hulme, 2012). Evidence from behavioural studies showed concurrent deficiencies in reading-related cognitive abilities, such as phonological awareness-namely, the representation, storage and retrieval of speech sounds (Ramus, Marshall, Rosen, \& Lely, 2013), verbal working memory (Szenkovits \& Ramus, 2005; Toffalini, Giofrè, \& Cornoldi, 2017), and rapid automatized naming (Di Filippo et al., 2005; Wolf, Bowers, \& Biddle, 2000). Phonological awareness deficit is widely considered the most influential cause of DD (for a review, see Vellutino, Fletcher, Snowling, \& Scanlon, 2004). Tallal and colleagues (Gaab, Gabrieli, Deutsch, Tallal, \& Temple, 2007; Tallal, Miller, \& Fitch, 1993a) identified a low-level auditory processing impairment as the basic cause of phonological deficits. According to the authors, individuals with DD show deficiencies in temporal resolution of rapidly changing auditory stimuli, and consequentially in speech perception. Therefore, an auditory-based remediation program for DD, designed to improve both dynamic auditory and phonological processing skills, called Fast ForWord, have been implemented by the authors (Merzenich et al., 1996; Tallal, 2004). Beneficial effects of the acoustic training on reading performances of children with DD have been reported, along with an increase of metabolic activity in left hemisphere temporoparietal language regions (Gaab et al., 2007; Temple et al., 2003). Rhythm perceptual abilities, namely, the accurate perception of metrical structure, has been found to be specifically impaired in DD as well (Corriveau \& Goswami, 2009; Goswami, Huss, Mead, Fosker, \& Verney, 2013; Huss, Verney, Fosker, Mead, \& Goswami, 2011). According to the 'Rise time hypothesis' (Goswami, 2011), sensitivity to metrical structure of speech in children with DD is affected by auditory perceptual impairments in sound rise time perception.

A typical oscillatory neural activity in individuals with DD has been proposed to have a casual role in the aforementioned phonological and auditory processing deficits (e.g., Giraud \& Ramus, 2013; Goswami, 2011). More precisely, auditory cortical oscillations of dyslexic individuals have been shown to entrain less accurately to the spectral properties of auditory stimuli at distinct frequency bands. 
Atypical phase-locking to slow (delta range) auditory nonverbal modulation in the right hemisphere have been observed in adults with DD (Hämäläinen, Rupp, Soltész, Szücs, \& Goswami, 2012) as well as a deficit in delta oscillatory neural response to natural speech stimuli occurs in poor readers (Abrams, Nicol, Zecker, \& Kraus, 2009). Recently, Lallier, Molinaro, Lizarazu, Bourguignon, and Carreiras (2017) proposed a cross-linguistic theoretical framework, in which two types of attentional-oscillatory mechanisms involved in the process of reading, operating in both the visual and auditory modalities, are described: (a) parsing mechanisms (i.e., spatiotemporal attentional shifting) in the delta and theta ranges, and their coupling, in charge of delimiting phonemic and orthographic chunks; (b) sampling mechanisms (i.e., attentional focus), in charge of identifying further the parsed chunks, via gamma-related activity. According to the authors, during reading the brain synchronization to low frequencies induces consistent attentional shifts between salient prosodic units, whereas the synchronization to high frequencies induces the sampling of the phonemic content within the focus of attention. In DD the deficit of low frequency neural entrainment leads to difficulties in sequential parsing of auditory information within the attentional focus, and consequentially to a poor analysis of prosodic syllabic contour of words and to a weak encoding of syllable positions within words.

Our hypothesis is that synchronizing speech prosody during reading with a regular acoustic stimulus could induce a more functional phaselocking at low frequency bands, inducing beneficial effects on temporal processing, and consequentially on reading. Accordingly, an intervention program for DD, called Rhythmic Reading Training (RRT) (Cancer, Bonacina, Lorusso, Lanzi, \& Antonietti, 2016), was devised. The program allows users to read out loud verbal stimuli synchronically with an external rhythmical sound, which is presented at a regular pace.

To maximize the effect of aforementioned behavioural training, we explored the possibility to combine it with a cortical neuromodulation technique, namely transcranial direct current stimulation (tDCS). Recent evidence showed that reading performance can be modulated using noninvasive brain stimulation by inducing excitability alterations in different brain regions involved in reading. Functional neuroimaging evidence suggested reduced left posterior temporal cortex (pTC) activity in individuals with DD (Maisog, Einbinder, Flowers, Turkeltaub, $\&$ Eden, 2008) and increased left pTC lateralization in children with DD after successful remedial training (Simos et al., 2002). Accordingly, Turkeltaub et al. (2012) improved words reading efficiency in below-average reading adults by enhancing left lateralization of pTC using tDCS, after just one session. Conversely, Thomson, Doruk, Mascio, Fregni, and Cerruti (2015) induced mild reading efficiency increase in normal reading adults using anodal tDCS over the right temporoparietal junction (CP6). Further, reading performance decrement was 
induced using left anodal stimulation over the homologue site (CP5). For this study, Thomson and colleagues used the contralateral mastoid for the reference site, whereas Turkeltaub and colleagues (2012) used the right hemisphere homologue (T8/TP8), resulting in cathodal flow directly at the right temporal parietal junction. Therefore, Turkeltaub and colleagues' results can be attributed by the conjunction of left anodal and right cathodal stimulation. A study involving adults with DD was carried out by Heth \& Lavidor (2015), who induced word-reading efficiency improvement using anodal tDCS over the left visual extrastriate area MT/V5, with the right orbito-frontal cortex as a reference site, for five sessions. Costanzo and colleagues were the first to study the effect of tDCS on young populations with DD. Firstly, the authors explored the optimal polarity of the stimulation for children and adolescents with DD in a single session intervention (Costanzo et al., 2016a). The authors found significant text reading accuracy improvement after left anodal/right cathodal tDCS and an increase in errors after left cathodal/right anodal tDCS. In a second study involving a group of Italian children and adolescent with DD, Costanzo and colleagues (2016a) used left anodal/right cathodal tDCS over temporoparietal regions in combination with a phonic training for 18 sessions. The intervention improved reading tasks specifically requiring phonological processing namely, pseudo-words reading speed and low frequency reading accuracy.

The aim of the present paper is to provide readers with an overview of the series of experimental trials which have been carried out to support the validity of RRT and to understand the specific conditions under which the training can be effective. New original results will be presented in detail, whereas results of already published studies will be summarised.

\section{THE RHYTHMIC READING TRAINING}

RRT is a computerized reading training program designed for Italian students with DD. RRT's main feature is the synchronization between reading fluency and an external acoustic stimulus, presented at a regular pace. Acoustic stimulation's pace is gradually increasing to improve reading fluency, along with the complexity of verbal stimuli presented. The training program comprises three categories of exercises, each aimed at training a specific reading ability: 'Syllables', 'Merging', and 'Words and Pseudo-words'. Users are taught to read out loud the verbal stimuli presented on the screen (i.e., syllables, words, pseudo-words, phrases) synchronically with the acoustic stimulation. During the first presentation of each exercise, the stimulus that is meant to be read is highlighted by a visual cue synchronized with the acoustic stimulation, 
so to make it easier for users to read the verbal material at a specific pace. The software allows the trainer to modulate the pace of the stimulation depending on each participant's reading level.

\section{RRT INDUCES IMPROVEMENT IN READING SKILLS HIGHER THAN THOSE OCCURRING THANKS TO NATURAL DEVELOPMENT}

Different studies with test-training-retest experimental design have been carried out to measure the effectiveness of RRT on reading speed and accuracy of Italian individual with DD. For all the studies reported here, participants had to fulfill the following inclusion criteria: (a) having been previously diagnosed with DD (ICD-10 code: F81.0) on the basis of standard inclusion and exclusion criteria (ICD-10: World Health Organization, 1992) and of the standard diagnosis procedure followed in Italy; (b) absence of comorbidity of DD and other neuropsychiatry or psychological conditions (whereas comorbidity with other learning disabilities was allowed); (c) not having been involved in any other intervention program for reading training at least in the past year.

The first study (Bonacina, Cancer, Lanzi, Lorusso, \& Antonietti, 2015) was conducted in a school setting and involved 28 junior high students with a diagnosis of DD, aged between 11 and 14. Participants were split into two groups matching gender, school grade, and level of reading impairment. The experimental group took part in an intervention using RRT for 9 biweekly training sessions of 30 minutes. The sessions were individual and supervised by a specialized trainer. As for the participants of the control group, they did not take part in any intervention during the experiment. Participants' reading performances were assessed before and after the training period using a battery of Italian standardized reading tests, which provide accuracy and speed scores for reading aloud text and lists of words and pseudo-words. Rhythm perception was also assessed using a rhythm reproduction task (Stambak, 1951) to check if RRT had an impact on this ability as well.

A mixed factorial ANOVA $(2 \times 2)$ was carried out to evaluate the effects of RRT on the reading measures collected. Results showed that participants who took part in RRT significantly improved their reading abilities, relative to those who did not take part in the intervention. Statistical significant differences were found in short and long pseudo-words reading speed, high frequency long words reading accuracy, and text reading accuracy.

These outcomes suggested that RRT induced greater changes in reading skills of students with DD relative to the natural development of reading efficiency. For those who participated in RRT, the gain in reading speed were 
averagely of +0.51 z-scores for short pseudo-words, and of +0.64 z-scores for long pseudo-words, whereas the gain in reading accuracy were averagely of +0.39 z-scores for high frequency long words, and of +2.37 z-scores for text. Concerning the rhythm reproduction task, no significant difference between the control and the intervention conditions was found. Therefore, the effect of RRT seemed to be specific on reading skills.

\section{THE EFFICACY OF RRT IS INDEPENDENT FROM MUSICAL EXPERTISE}

The second study investigated the difference in RRT efficacy depending on the music expertise of the participants. To do that, ten 11-14 y.o. junior highschool students were involved in a training program using RRT for 10 weeks. For this study, the sessions occurred once a week and lasted 30 minutes each. Half of the participants were at that time enrolled in a music course and practiced a musical instrument (i.e., guitar, piano, or drums), whereas the other half had never been involved in any music activity. Reading skills of both groups were enhanced by RRT. Pre- and post-training performances of all participants were compared using Wilcoxon signed-rank test. Significant gains occurred in reading speed of short pseudo-words $(\mathrm{z}=-2.67 ; \mathrm{p}<.01 ; \mathrm{r}=.59)$, long pseudo-words $(\mathrm{z}=-2.67 ; \mathrm{p}<.01 ; \mathrm{r}=.60)$, and for low frequency short words $(\mathrm{z}=-2.17 ; \mathrm{p}<.05 ; \mathrm{r}=.49)$. Finally, low frequency short words reading accuracy significantly improved $(\mathrm{z}=-2.37 ; \mathrm{p}<.05 ; \mathrm{r}=.53)$.

Interestingly, no differences in reading improvements were found between the two groups for any of the reading parameters assessed. Hence music expertise did not play a role in the effectiveness of RRT (see Fig. 1). These findings suggested that both individuals with DD who are expert in music and those who are not can benefit from RRT to a similar extent. 


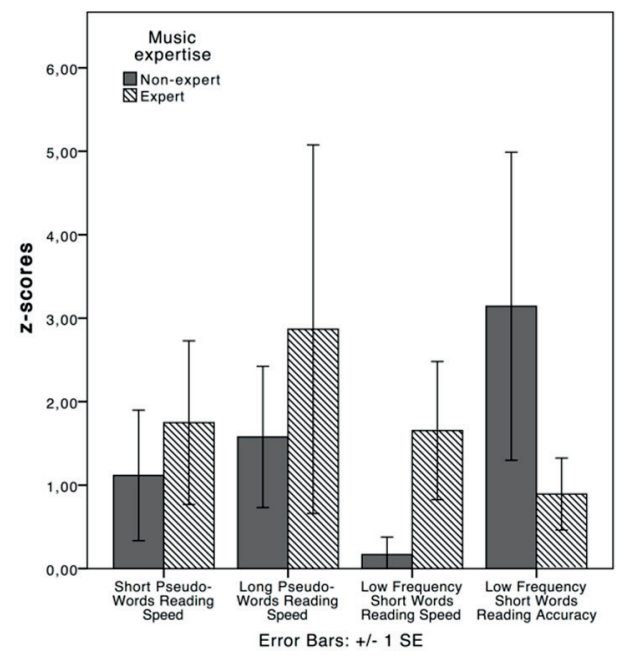

Figure 1. Reading gains (expressed in z-scores) after RRT in music experts and nonexperts

\section{IMPLEMENTING RRT IN CLINICAL SETTINGS}

The two studies mentioned previously were carried out in settings (a school and a music training centre) where rehabilitations treatments were not usually delivered and participants were asked to serve as volunteers in an experimental design. The third study aimed at testing the applicability of RRT in a clinical setting, namely, in the Childhood and Adolescence Neuropsychiatry Unit of an hospital (Cancer, Manzoli, \& Antonietti, 2016). This kind of hospital departments are the environment where most commonly interventions for learning disabilities are delivered in the Italian context. Participants were recruited among the Neuropsychiatry Unit's patients who were part of the waiting list for an intervention targeting on learning disabilities. Seven primary and junior high school students aged 9-11 $(\mathrm{M}=9.77$; $\mathrm{SD}=0.71)$ with a diagnosis of $\mathrm{DD}$ in comorbidity with at least another learning disability (i.e., Dysorthography, Dysgraphia, Dyscalculia) took part in an intervention using RRT. The sessions occurred twice a week for 10 weeks and lasted 20 minutes each. Straight after each RRT session, participants were involved for further 20 minutes in a traditional intervention, delivered by a speech therapist of the Unit, targeting on the other learning disabilities but DD. Besides reading, other neuropsychological 
functions involved in or related to the reading process (i.e., visual sustained attention, auditory selective attention, verbal working memory, and rhythm perception abilities) were assessed before and after the intervention period. The extent of the reading gains was averagely slightly lower and showed greater variability, relative to the previous studies $(+0.21 \pm 0.33 \mathrm{z}$-scores in pseudo-words reading speed; $+0.17 \pm 0.28 \mathrm{z}$-scores in text reading speed; $+0.57 \pm 1.01$ in pseudo-words reading accuracy; $+0.65 \pm 1.36 \mathrm{z}$-scores in text reading accuracy), likely because more than one learning process was involved in the intervention (see Fig. 2). Therefore, it is preferable not to deliver RRT in a mixture of methodologies targeting different learning disabilities. Concerning the notable variability in reading gains, we argue that the small sample size, along with an initial great variability in participant's baseline measures, induced a more variable outcome. As for the other cognitive measures, increases in z-scores occurred for visual sustained attention $(+0.83 \pm 0.88 \mathrm{z}$-scores $)$ and verbal working memory (forward digit span: $+0.26 \pm 0.44 \mathrm{z}$-scores). These abilities are indeed greatly involved in the performance of RRT exercises.

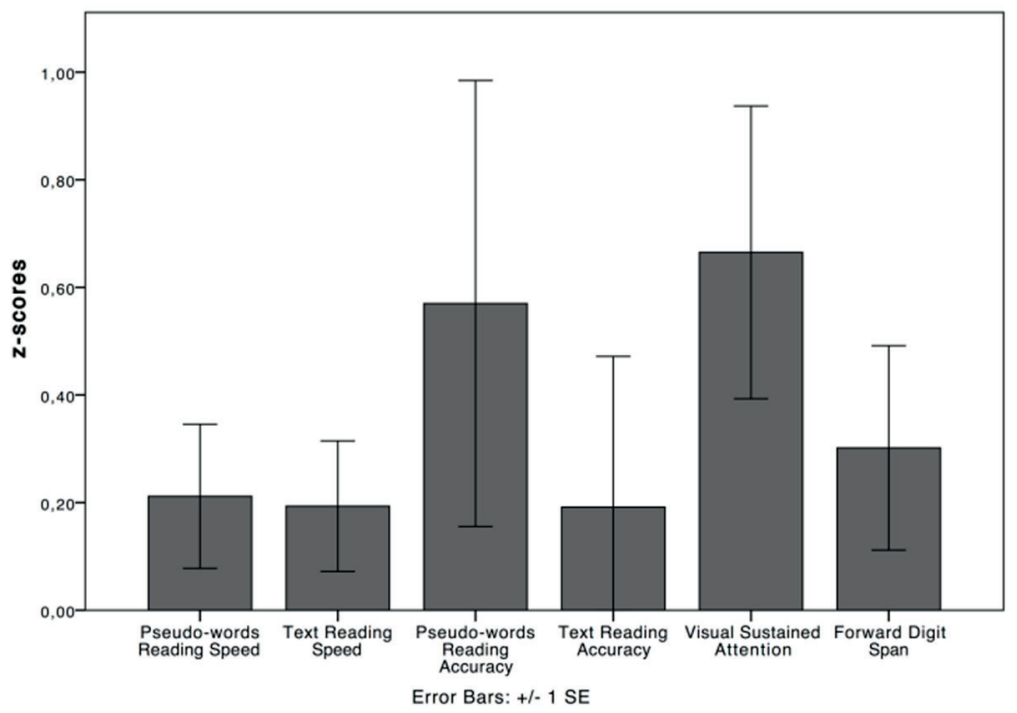

Figure 2. Reading, attention, and verbal working memory gains (expressed in $z$-scores) after RRT delivered in a clinical setting 


\section{COMPARING THE EFFICACY OF RRT WITH OTHER TREATMENTS}

After having confirmed that (1) RRT enhances reading skills more than natural development (first study), (2) all students with DD can benefit from RRT regardless of music expertise (second study), and (3) RRT is applicable in a clinical rehabilitation setting, preferably delivered alone (third study), it was relevant to compare the impact of RRT with other interventions for DD.

\subsection{RRT compared with hemisphere-specific stimulation and action video games training}

For the first comparative study, a remediation package consisting of the combination of two training methods for DD, both documented in their effectiveness when used separately, was chosen. This package comprised (a) a treatment devised after Bakker's Balance Model (Bakker \& Licht, 1986) and known as Visual Hemisphere-Specific Stimulation (VHSS) (Lorusso, Facoetti, \& Bakker, 2011; Lorusso, Facoetti, Paganoni, Pezzani, \& Molteni, 2006), that is, the most effective treatment for DD in the Italian setting according to Tressoldi, Vio, Lorusso, Facoetti, and Iozzino, 2003 and (b) a recent proposed intervention which consists in playing action-videogames (AVG) (Franceschini et al., 2013). The aim of VHSS is to draw the less involved hemisphere, according to Bakker's model (Bakker \& Licht, 1986), into the reading process by presenting words tachistoscopically and selectively to a visual hemifield using a computerized program. As for AVG training, it involves the participants in a video-game training by Nintendo Wii technology, specifically aimed at improving visuospatial attentional abilities, that are crucial for the perception of stimuli during reading (Franceschini, Gori, Ruffino, Pedrolli, \& Facoetti, 2012).

Twenty-two primary and junior high-school students with a diagnosis of DD aged between 8 and $12(\mathrm{M}=9.59, \mathrm{SD}=1.56)$, recruited among patients of a Neuropsychiatry Unit, were involved in the study (Cancer et al., submitted). Two subgroups of the same size matched for gender, age, IQ, dyslexia subtype (classified according to the Balance Model), and level of reading impairment were assigned to either RRT or an intervention resulting from the combination of VHSS and AVG. The intervention schedule consisted of 18 sessions over 9 days (two 45-minute sessions per day), conducted by experts in the use of each methodology. Participants' reading abilities and a set of neuropsychological functions (i.e., phonological awareness, auditory selective attention, and rhythm perception abilities) were assessed before and after the involvement in the intervention.

Pre-post training comparisons (Wilcoxon signed-rank test) showed a significant improvement in all reading and phonological measures in both VHSS + AVG and RRT groups. No significant differences were found between the two 
experimental conditions, therefore both interventions significantly improved participants' reading and phonological skills to a similar extent. RRT induced greater effects on reading speed and VHSS-AVG produced greater effects on reading accuracy, although the differences between the two kinds of intervention were not statistically significant. Similarly to the first and third study, RRT did not induce significant improvements in rhythm perception abilities, confirming its specific effectiveness on reading. The fact that RRT alone had a similar impact on reading than of a multimodal intervention package involving two validated techniques, adduced crucial evidence supporting RRT efficacy for DD.

\subsection{RRT compared with Sublexical and Lexical Recognition Automatization training}

In the attempt to find the best RRT intervention protocol for maximizing its potential, another comparative study was carried out. Since one of the previous study (namely, the third) suggested that presenting RRT in combination with other techniques was slightly less efficient, for the present study the intervention involved RRT only. Its effectiveness was therefore compared with another intervention for DD, which comprises several validated treatments resulting in a proposal tailored to meet individual needs and features: Sublexical and Lexical Recognition Automatization training (SLRA). The aim of SLRA is to train the recognition and decoding of sublexical and lexical stimuli, towards an automatization. Sixteen 8 to 11 y.o. participants $(M=9.35 ; S D=0.82)$ took part in the fifth study. Two subgroups matched for gender, age, and level of reading impairment were assigned either to RRT or SLRA intervention group. As for those involved in RRT, the procedure comprised 10 biweekly sessions of 60 minutes each, whereas for SLRA 10 weekly sessions under the supervision of a trainer, plus 60 minutes of homework assignments once a week, using materials provided by the trainer. Significant pre-post training differences in reading speed (Wilcoxon signed-rank test) occurred in both conditions. SLRA induced significant improvements in words $(\mathrm{z}=-2.20 ; p<.05 ; r=.55 ; \mathrm{Mdn}=0.33)$ and text $(\mathrm{z}=-2.37 ; p<.05 ; r=.60 ; \mathrm{Mdn}=0.22)$ reading, whereas RRT improved words $(\mathrm{z}=-2.55 ; p<.05 ; r=.63 ; \mathrm{Mdn}=0.62)$, pseudo-words $(\mathrm{z}=-2.67 ; p<$ $.005 ; r=.66 ; \mathrm{Mdn}=0.83)$, and text $(\mathrm{z}=-1.95 ; p=.05 ; r=.48 ; \mathrm{Mdn}=0.21)$ reading (see Fig. 3). Further, a significant difference (Mann-Whitney test) in pseudo-words reading speed $(U=1.00 ; p=.001 ; r=.81)$ emerged between the two interventions, for which RRT was more effective. 


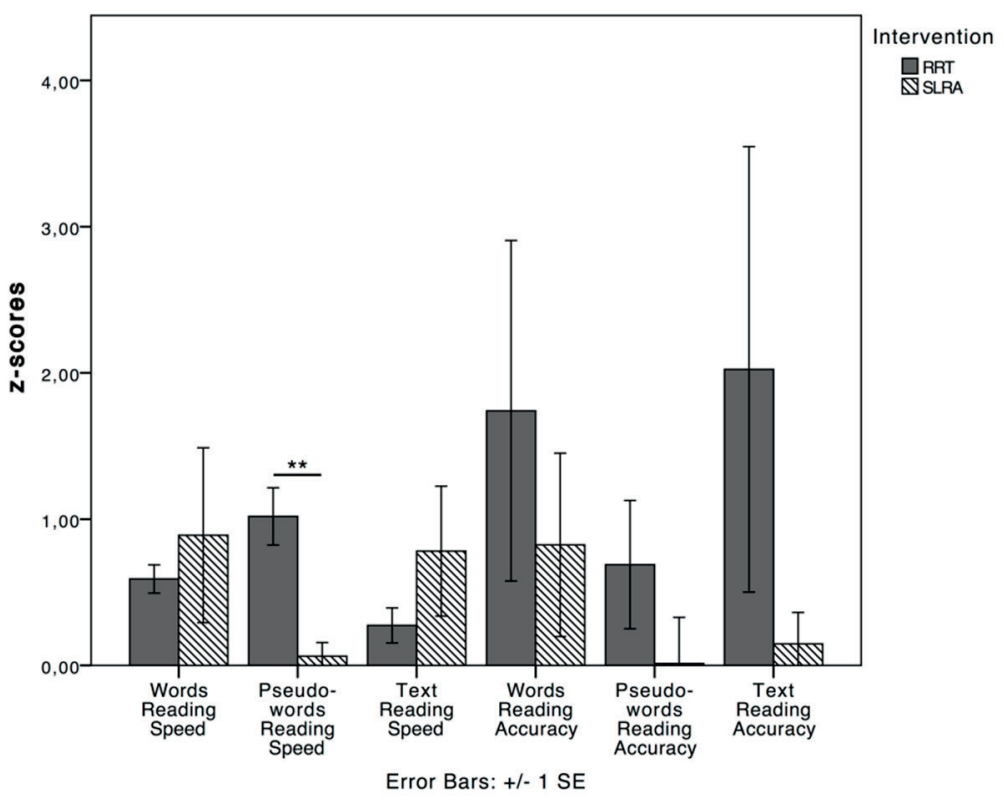

Figure 3. Reading gains differences (expressed in z-scores) between RRT and Sublexical and Lexical Recognition Automatization training conditions

These results showed that RRT induced benefits comparable (and greater for words reading speed) to those of an intervention involving several training materials and extra assignments that the family of the child has to manage at home.

\section{COMBINING RRT WITH AN AUDITORY TRAINING}

Although the previous five studies' results were encouraging, there were several questions about the effectiveness of RRT still unanswered, namely: (a) Are reading improvements induced by RRT maintained over time? (b) Is it possible to induce even greater effects on reading by combining RRT with another intervention that shares the same theoretical background? (c) Is there a relationship between reading improvements induced by RRT and the potential effects of the training on other 
neuropsychological functions related to reading? (d) Do the ones who initially have lower reading skills benefit from RRT to a greater extent?

A correlational study was carried out to address those points. Nineteen participants aged 8-14 $(\mathrm{M}=10.77 ; \mathrm{SD}=1.35)$ were recruited among patients of Neuropsychiatry Units. All of them were enrolled in an intervention resulting from the combination of RRT and a specific auditory training. The latter involved several music games (Antonietti et al., 2017) specifically designed for improving auditory processing abilities typically compromised in children with DD (for a review, see Hämäläinen, Salminen, \& Leppänen, 2013) and at the same time providing an active engagement with music. The intervention was delivered for a total of 20 hours over 10 weeks (60 minutes biweekly sessions). Participants' reading abilities and the same set of neuropsychological functions related to reading from study three (i.e., visual sustained attention, auditory selective attention, verbal working memory, and rhythm perception abilities) were assessed before and after the intervention. Further, a subgroup of 8 participants were involved in a follow-up assessment three months after the end of the training period.

Regarding the efficacy of the combined intervention (question a), significant positive effects on reading speed were confirmed (words: $\mathrm{z}=-$ 3.01, $p<.005, r=.49$; pseudo-words: $\mathrm{z}=-3.55, p<.0001, r=.49$; text: $\mathrm{z}=$ $-3.29, p=.001, r=.53$ ) (see Fig. 4).

As for question $b$, no significant differences emerged between the postand follow-up measures for all the functions assessed. Therefore, reading gains induced by RRT were maintained for three months after the intervention. Moreover, the comparisons between pre- and follow-up performances differed significantly in reading speed (words: $\mathrm{z}=-2.52, r=-.89, p=.01$; pseudowords: $\mathrm{z}=-2.10, r=-.74, p<.05$; text: $\mathrm{z}=-2.52, r=-.89, p=.01)$ and accuracy (words: $\mathrm{z}=-2.41, r=-.85, p<.05$; pseudo-words: $\mathrm{z}=-2.53, r=-$ $.89, p=.01)$. The effects on reading accuracy appeared to have been consolidated after the end of the intervention. As for the subgroup of participants taking part in the follow-up assessment, reading performances were still significantly improved three months after the end of the intervention, relative to baseline measures (see Fig. 5). These differences adduced crucial evidence to the medium-term efficacy of the intervention. 


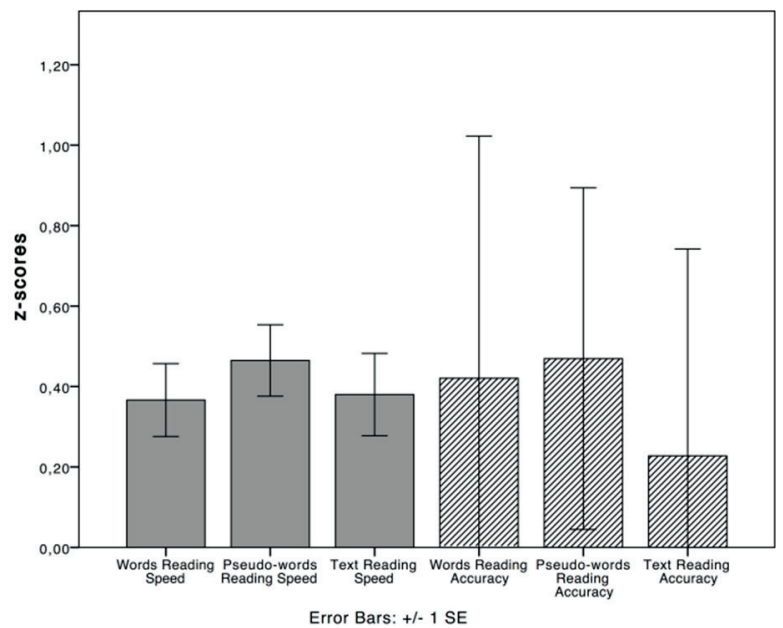

Figure 4. Reading speed (grey bars) and reading accuracy (striped bars) gains (expressed in z-scores) after RRT in combination with specific auditory training

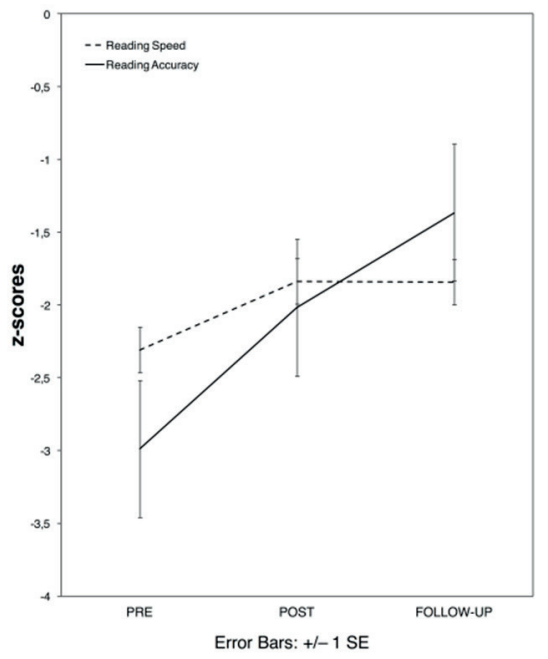

Figure 5. Pre, post, and follow-up global measures (expressed in z-scores) of reading speed (dotted line) and reading accuracy (solid line) in a subgroup of participants who took part in $R R T+$ specific auditory training 
Concerning the other reading-related variables (question c), significant prepost training differences were found in auditory attention $(\mathrm{z}=-2.61 ; \mathrm{p}<.05 ; \mathrm{r}=$ $.42)$, rhythm reproduction $(\mathrm{z}=-2.91 ; \mathrm{p}<.005 ; \mathrm{r}=.47)$, and visual sustained attention $(\mathrm{z}=-3.82 ; \mathrm{p}<.0001 ; \mathrm{r}=.62)$. As well as for the reading measures, these gains were found to be similar at the follow-up assessment. To better understand these effects, non-parametric correlations (Spearman's correlations) were computed between reading improvements and reading-related variables improvements. As predictable, reading speed measures increases were positively correlated between each other, namely words and pseudo-words reading speed (rs $=.725 ; \mathrm{p}<.0001)$, words and text reading speed $(\mathrm{rs}=.535 ; \mathrm{p}<.05)$, and pseudo-words and text reading speed ( $\mathrm{rs}=.67 ; \mathrm{p}<.005)$. Interestingly, no correlations between reading improvements and neuropsychological measures improvements were found. Finally, several negative correlations between baseline skills and reading gains after the intervention emerged (question d), more precisely between pre-training backword digit span and pseudo-words reading speed enhancement ( $r s=-.66 ; \mathrm{p}<.005$ ), between pre-training pseudo-words accuracy and pseudo-words and text accuracy improvements (respectively, $\mathrm{rs}=-$ $.58 ; \mathrm{p}<.01$ and $\mathrm{rs}=-.67 ; \mathrm{p}<.005)$, and between pre-training text accuracy and text accuracy gains (rs $=-.79 ; \mathrm{p}<.0001)$.

In conclusion, (a) the combination of RRT with a specific auditory training was found to be slightly less effective than RRT only in enhancing reading skills; (b) The improvements induced by RRT were maintained several months after the intervention's ending; (c) Even though significant gains occurred in auditory attention, visual attention, and rhythm reproduction abilities, they did not correlate with reading gains; (d) Participants who had worse verbal working memory before the intervention benefited more in terms of reading speed and those who struggled the most with reading accuracy showed the greatest gains in this parameter.

\section{COMBINING RRT WITH NEUROMODULATION IN UNDERGRADUATE STUDENTS WITH DD}

After studying the potential of RRT on children and pre-adolescents with DD, we were interested in broadening the possibilities of its application by targeting a population not usually involved in any intervention for enhancing reading, namely, adults with DD. For doing so, few pilot applications involving a small group of undergraduate students with a diagnosis of DD have been carried out. These applications were aimed at testing the applicability of RRT's suitably modified version for adults. Encouraging preliminary results emerged, concerning both 
reading improvements and participants' engagement and interest. Therefore, a seventh study was designed. Along with RRT, the study protocol involved neuromodulation, namely, tDCS, to maximize the effect of the intervention. It has been indeed suggested that performance gains induced by a behavioral training can be maximized when combined with cortical neuromodulation techniques, such as tDCS, which increase neuroplasticity (Vicario \& Nitsche, 2013).

The present ongoing study involved undergraduate students with a diagnosis of DD in an intervention comprising RRT and tDCS for 10 daily sessions over 2 weeks. No participants were taking any central nervous systemactive drugs, neither had a history or presence of any neurological or psychiatric disorder. For safety reasons regarding the tDCS procedure, potential participants were also screened for pregnancy, seizures, epilepsy, severe headaches, and metallic, electrically sensitive or mechanical implanted devices. The study is meant to have a sham-controlled parallel design. Therefore, participants have been pseudo-randomly assigned to either the active or sham tDCS condition. Direct current was generated by HDC Series stimulator by Newronika (Milan) and delivered by a pair of two $5 \times 5 \mathrm{~cm}\left(25 \mathrm{~cm}^{2}\right.$ each) rubber electrodes enveloped in saline-soaked sponges covered with conductive gel. The anodal electrode was positioned over the participants' left temporoparietal region, on the area corresponding midway between P7 and TP7, according to the 10-20 EEG international system. The cathodal electrode was positioned over the homologue ipsilateral region (midway between P8 and TP8). This specific montage has been used by Costanzo et al. in their intervention protocols (2016a; 2016b). The left area was stimulated at a constant current of $1.5 \mathrm{~mA}$ for 20 minutes, resulting in a current density of $0.06 \mathrm{~mA} / \mathrm{cm}^{2}$. At the beginning of stimulation, the current was gradually increased (ramp-up) in 7 proportional intervals during the first 7 seconds to the stimulation threshold. Stimulation was initiated 5 minutes before the beginning of RRT. RRT exercises were delivered for 30 minutes and presented simultaneously with the stimulation for the initial 15 minutes. For both active and sham conditions, pre-post assessment reading measures were collected, as well as other cognitive functions involved in the process of reading, namely, lexical access (rapid automatized naming, RAN), verbal working memory (forward and backwards digit span), and rhythm perception abilities. Four weeks after the end of the intervention, participants took part in a follow-up assessment in order to test the maintenance of the improvements. Regarding tDCS tolerability, adverseeffects were assessed after each stimulation session using a standard questionnaire (Brunoni et al., 2011) listing symptoms such as tingling, itching, burning sensation, headache, and scalp pain.

Here are presented preliminary results from three single cases, who took part in the active tDCS condition. No participant reported significant 
discomfort at the electrode sites during or after the stimulation. However, mild tingling, itching, and burning sensation were sometimes reported, but usually vanished quickly after habituation.

Case 1 is a 23 y.o. male university student enrolled in the 4th year of Economics course, with a diagnosis of DD in comorbidity with Dysorthography. His baseline reading abilities were compromised both for accuracy and speed. After taking part in 10 sessions of RRT and active tDCS, his global reading accuracy was enhanced of $+1.99 \mathrm{z}$-scores and his global reading speed improved of $+0.40 \mathrm{z}$-scores (computing the means of words, pseudo-words and text reading efficiency). These gains were maintained 4 weeks after the end of the intervention, with a slight decrease in accuracy, which however did not reach the baseline (see Fig. 6).

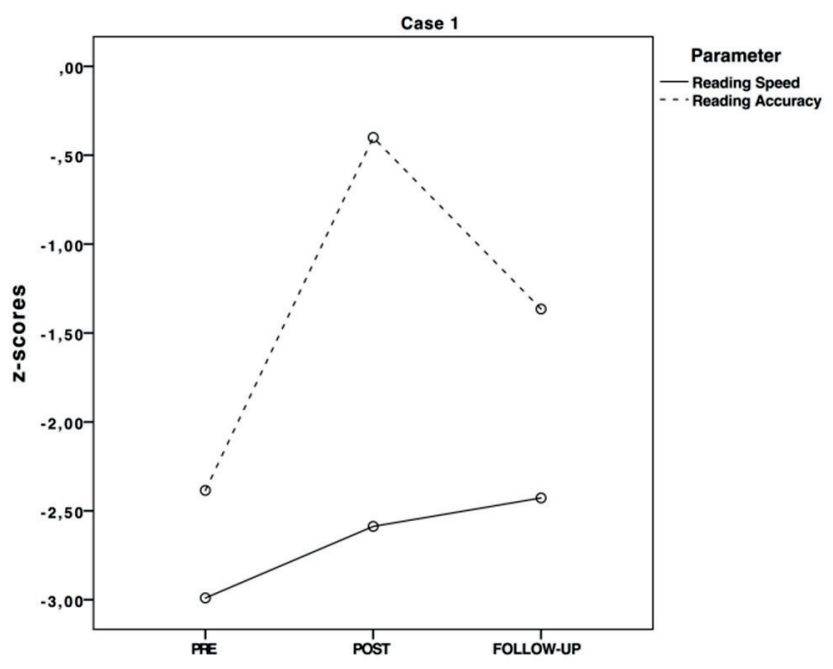

Figure 6. Case 1 pre, post, and follow-up global measures (expressed in z-scores) of reading speed (solid line) and global reading accuracy (dotted line)

Case 2 is a female 19 y.o. Educational Sciences undergraduate (1st year), who has a diagnosis of DD in comorbidity with Dysorthography and Dyscalculia. The reading parameter in which she struggled the most at baseline was speed, whereas reading accuracy was similar to the norm. The intervention 
induced gains in both reading speed and accuracy, respectively of +0.31 and +0.13 . These gains were maintained at follow-up (see Fig. 7).

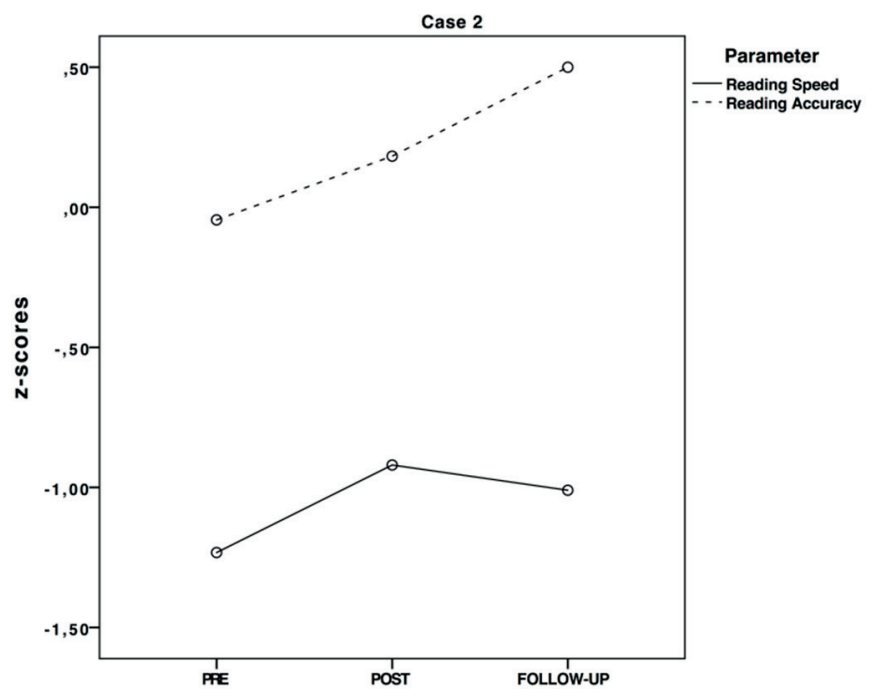

Figure 7. Case 2 pre, post, and follow-up global measures (expressed in z-scores) of reading speed (solid line) and global reading accuracy (dotted line)

Case 3 is a 26 y.o. undergraduate at his last year of Statistics course, with a diagnosis of DD only. At baseline, his reading speed was greatly compromised (6.88 z-scores below the norm), whereas his reading was accurate. After the intervention, reading speed improved of $4.08 \mathrm{z}$-scores. Such increase in speed was compensated by a reading accuracy decrement. Reading speed increase was maintained a month after the end of the intervention and reading accuracy slightly improved relative to post-training assessment (see Fig. 8).

Single cases' preliminary results provided encouraging evidence about the efficacy of RRT on adults with DD. However, the effects seem to depend greatly on individual reading profile at baseline. Concerning tDCS, its impact will be measure by the comparison between active and sham condition. 


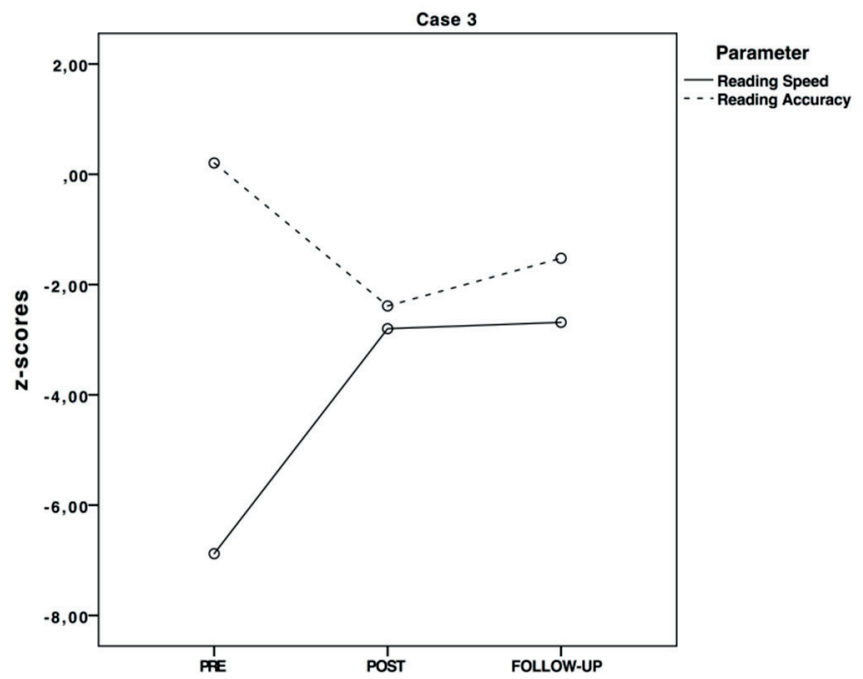

Figure 8. Case 3 pre, post, and follow-up global measures (expressed in z-scores) of reading speed (solid line) and global reading accuracy (dotted line)

\section{CONCLUSIONS}

In the present paper we presented an overview of several trials carried out to support the validity of RRT as a remedial intervention for DD. The training was designed according to the evidence of a deficit in temporal processing of acoustic stimuli in DD (e.g., Tallal, Miller, \& Fitch, 1993b) that depends on deficiencies in low frequency neural entrainment to acoustic features of sounds (for a review, see Lallier et al., 2017).

Firstly, we verified that RRT was able to induce greater improvements in reading skills of junior high-school students with DD relative to natural development. Secondly, we showed that musical expertise is not a necessary condition for benefitting from RRT in terms of reading gains. Hence, the intervention is suitable for those who do not have any musical aptitude as well. The third study, carried out in a clinical rehabilitation setting, confirmed the efficacy of RRT on reading skills, as well as on other reading-related neuropsychological variables, namely, visual sustained attention and verbal working memory. However, this study highlighted RRT potential greater efficacy when delivered alone, relative to combining it with other interventions 
targeting different learning difficulties. After that, two comparative studies have been carried out. The study demonstrated that RRT is as effective as a multimodal intervention package involving two validated techniques, that is, Hemisphere-Specific Stimulation (Lorusso et al., 2011) and Action Video Games Training (Franceschini et al., 2013). Further, a tendency of RRT to induce greater effects on reading speed and of the multimodal package to induce greater effects on reading accuracy was observed. The second comparative study matched RRT with another multimodal intervention, namely, SLRA which comprises several training materials and extra-home assignments. Consistently with the previous comparative study, RRT induced benefits comparable (and greater for words reading speed) to those of the multimodal intervention. The efficacy of RRT combined with an auditory training specifically designed for improving acoustic processing was tested afterwards. This combination was found to be slightly less effective than RRT only in enhancing reading skills. However, the induced improvements were maintained several months after the end of the intervention. Significant improvements in auditory attention, visual attention, and rhythm reproduction abilities emerged as well, but those improvements did not correlate with reading gains. It was clarified that participants who had worse verbal working memory before the intervention benefited more in terms of reading speed and those who struggled the most with reading accuracy showed the greatest gains in this parameter. Finally, an ongoing study is exploring the possibility to maximize RRT efficacy by combining it with a neuromodulation technique, namely, tDCS. For this last study, an older population of students with DD, namely undergraduates, was involved. Single cases preliminary results provided encouraging evidence about RRT and active tDCS efficacy on adults with DD.

Overall, RRT was found to be an effective intervention for DD across different ages and despite individual characteristics (e.g., musical expertise, level of reading impairment). Even though RRT induced significant improvements in both reading speed and reading accuracy, the greatest and most consist effects were found on reading speed. The temporal feature of RRT, implemented through the constant stress on pace increase, makes it indeed particularly suitable to enhance reading speed. Further, the fact that RRT's effectiveness was comparable with that of validated multimodal approaches is promising, especially considering its simplicity. RRT is in fact easy to use and it does not need a long practice to be managed. It requires neither a specific setting nor specific technology (besides a computer with speakers), nor homework assignments. Further, its rhythmical feature makes it more appealing and less tedious for users, relative to traditional intervention for improving reading. Regarding all the studies presented in this paper, no participant ever dropped out. We suggest that the music component of the intervention was salient enough to provide 
attentional focus and motivation during the training period. Moreover, we argue that such innovating approach, comprising both reading and rhythm, could have exploited the creative potential of individuals with DD, who were found to be more creative relative to their normal reading peers (e.g., Antonietti \& Cancer, 2016; Cancer, Manzoli, \& Antonietti, 2016).

\section{REFERENCES}

Abrams, D. A., Nicol, T., Zecker, S., \& Kraus, N. (2009). Abnormal Cortical Processing of the Syllable Rate of Speech in Poor Readers. Journal of Neuroscience, 29(24), 7686-7693.

Antonietti, A., \& Cancer, A. (2016). La creatività come risorsa per gli studenti con dislessia. Psicologia E Scuola, 44, 50-57.

Antonietti, A., Colombo, A., Germagnoli, S., Pace, G., Pradella, C., \& Stievano, G. (2017). A suon di lettere. Attività sonoro-musicali per il potenziamento delle abilità linguistiche. Milano: Rugginenti.

Bakker, D. J., \& Licht, R. (1986). Learning to read: Changing horses in midstream. In G.T. Pavlidis \& D.F. Fisher, Dyslexia: its neuropsychology and treatment. New York City: Wiley.

Bonacina, S., Cancer, A., Lanzi, P. L., Lorusso, M. L., \& Antonietti, A. (2015). Improving reading skills in students with dyslexia: the efficacy of a sublexical training with rhythmic background. Frontiers in Psychology, 6, 1-8.

Brunoni, A. R., Amadera, J., Berbel, B., Volz, M. S., Rizzerio, B. G., \& Fregni, F. (2011). A systematic review on reporting and assessment of adverse effects associated with transcranial direct current stimulation. International Journal of Neuropsychopharmacology, 14(8), 1133-1145.

Cancer, A., Bonacina, S., Antonietti, A., Salandi, A., Molteni, M., \& Lorusso, M. L. (submitted). Rhythmic Reading Training compared with Hemisphere-Specific Stimulation and Action Video Games: a pseudo-randomized controlled trial. PLOS ONE.

Cancer, A., Bonacina, S., Lorusso, M. L., Lanzi, P. L., \& Antonietti, A. (2016). Rhythmic reading training (RRT): A computer-assisted intervention program for dyslexia. In S. Serino, A. Matic, D. Giakoumis, G. Lopez, \& P. Cipresso (Eds.), Pervarsive computing paradigms for mental health. Communications in Computer and Information Science, 604, (pp. 249258). Switzerland: Springer International Publishing. 
Cancer, A., Manzoli, S., \& Antonietti, A. (2016). The alleged link between creativity and dyslexia: Identifying the specific process in which dyslexic students excel. Cogent Psychology, 3(1), 1190309.

Corriveau, K. H., \& Goswami, U. (2009). Rhythmic motor entrainment in children with speech and language impairments: Tapping to the beat. Cortex, 45(1), 119-130.

Costanzo, F., Varuzza, C., Rossi, S., Sdoia, S., Varvara, P., Oliveri, M., ... \& Menghini, D. (2016b). Evidence for reading improvement following tDCS treatment in children and adolescents with Dyslexia. Restorative Neurology and Neuroscience, 34(2), 215-226.

Costanzo, F., Varuzza, C., Rossi, S., Sdoia, S., Varvara, P., Oliveri, M., ... \& Menghini, D. (2016a). Reading changes in children and adolescents with dyslexia after transcranial direct current stimulation: NeuroReport, 1.

Di Filippo, G., Brizzolara, D., Chilosi, A., Luca, M. D., Judica, A., Pecini, C., ... \& Zoccolotti, P. (2005). Rapid naming, not cancellation speed or articulation rate, predicts reading in an orthographically regular language (Italian). Child Neuropsychology, 11(4), 349-361.

Franceschini, S., Gori, S., Ruffino, M., Pedrolli, K., \& Facoetti, A. (2012). A Causal Link between Visual Spatial Attention and Reading Acquisition. Current Biology, 22(9), 814-819.

Franceschini, S., Gori, S., Ruffino, M., Viola, S., Molteni, M., \& Facoetti, A. (2013). Action Video Games Make Dyslexic Children Read Better. Current Biology, 23(6), 462-466.

Gaab, N., Gabrieli, J. D. E., Deutsch, G. K., Tallal, P., \& Temple, E. (2007). Neural correlates of rapid auditory processing are disrupted in children with developmental dyslexia and ameliorated with training: an fMRI study. Restorative Neurology and Neuroscience, 25, 295-310.

Giraud, A. L., \& Ramus, F. (2013). Neurogenetics and auditory processing in developmental dyslexia. Current Opinion in Neurobiology, 23(1), 37-42.

Goswami, U. (2011). A temporal sampling framework for developmental dyslexia. Trends in Cognitive Sciences, 15(1), 3-10.

Goswami, U., Huss, M., Mead, N., Fosker, T., \& Verney, J.P. (2013). Perception of patterns of musical beat distribution in phonological developmental dyslexia: Significant longitudinal relations with word reading and reading comprehension. Cortex, 49(5), 1363-1376.

Hämäläinen, J. A., Rupp, A., Soltész, F., Szücs, D., \& Goswami, U. (2012). Reduced phase locking to slow amplitude modulation in adults with dyslexia: An MEG study. NeuroImage, 59(3), 2952-2961. 
Hämäläinen, J. A., Salminen, H. K., \& Leppänen, P. H. T. (2013). Basic Auditory Processing Deficits in Dyslexia: Systematic Review of the Behavioral and EventRelated Potential / Field Evidence. Journal of Learning Disabilities, 46(5), 413-427.

Heth, I., \& Lavidor, M. (2015). Improved reading measures in adults with dyslexia following transcranial direct current stimulation treatment. Neuropsychologia, 70, 107-113.

Huss, M., Verney, J. P., Fosker, T., Mead, N., \& Goswami, U. (2011). Music, rhythm, rise time perception and developmental dyslexia: Perception of musical meter predicts reading and phonology. Cortex, 47(6), 674-689.

Lallier, M., Molinaro, N., Lizarazu, M., Bourguignon, M., \& Carreiras, M. (2017). Amodal Atypical Neural Oscillatory Activity in Dyslexia: A CrossLinguistic Perspective. Clinical Psychological Science, 5(2), 379-401.

Lorusso, M. L., Facoetti, A., \& Bakker, D. J. (2011). Neuropsychological Treatment of Dyslexia: Does Type of Treatment Matter? Journal of Learning Disabilities, 44(2), 136-149.

Lorusso, M. L., Facoetti, A., Paganoni, P., Pezzani, M., \& Molteni, M. (2006). Effects of visual hemisphere-specific stimulation versus reading-focused training in dyslexic children. Neuropsychological Rehabilitation, 16(2), 194-212.

Maisog, J. M., Einbinder, E. R., Flowers, D. L., Turkeltaub, P. E., \& Eden, G. F. (2008). A Meta-analysis of Functional Neuroimaging Studies of Dyslexia. Annals of the New York Academy of Sciences, 1145(1), 237-259.

Merzenich, M. M., Jenkins, W. M., Johnston, P., Schreiner, C., Miller, S. L., \& Tallal, P. (1996). Temporal Processing Deficits of Language-Learning Impaired Children Ameliorated by Training. Science, 271(5245), 77-81.

Ramus, F., Marshall, C. R., Rosen, S., \& Lely, H. K. J. van der Lely, H. K. (2013). Phonological deficits in specific language impairment and developmental dyslexia: towards a multidimensional model. Brain, 136(2), 630-645.

Richlan, F. (2014). Functional neuroanatomy of developmental dyslexia: the role of orthographic depth. Frontiers in Human Neuroscience, 8, 347.

Simos, P. G., Fletcher, J. M., Bergman, E., Breier, J. I., Foorman, B. R., Castillo, E. M., ... \& Papanicolaou, A. C. (2002). Dyslexia-specific brain activation profile becomes normal following successful remedial training. Neurology, 58(8), 1203-1213.

Snowling, M. J., \& Hulme, C. (2012). Annual Research Review: The nature and classification of reading disorders - a commentary on proposals for DSM5. Journal of Child Psychology and Psychiatry, 53(5), 593-607.

Stambak, M. (1951). Le problème du rythme dans le développement de l'enfant et dans les dyslexies d'évolution. Enfance, 4(5), 480-502. 
Szenkovits, G., \& Ramus, F. (2005). Exploring dyslexics' phonological deficit I: lexical vs sub-lexical and input vs output processes. Dyslexia, 11(4), 253-268.

Tallal, P., Miller, S., \& Fitch, R. H. (1993a). Neurobiological basis of speech: a case for the preeminence of temporal processing. Annals of the New York Academy of Sciences, 682, 27-47.

Tallal, P., Miller, S., \& Fitch, R. H. (1993b). Neurobiological Basis of Speech: A Case for the Preeminence of Temporal Processing. Annals of the New York Academy of Sciences, 682(1), 27-47.

Tallal, P. (2004). Improving language and literacy is a matter of time. Nature Reviews. Neuroscience, 5(9), 721-728.

Temple, E., Deutsch, G. K., Poldrack, R. A, Miller, S. L., Tallal, P., Merzenich, M. M., \& Gabrieli, J. D. E. (2003). Neural deficits in children with dyslexia ameliorated by behavioral remediation: evidence from functional MRI. Proceedings of the National Academy of Sciences of the United States of America, 100(5), 2860-2865.

Thomson, J. M., Doruk, D., Mascio, B., Fregni, F., \& Cerruti, C. (2015). Transcranial direct current stimulation modulates efficiency of reading processes. Frontiers in Human Neuroscience, 9.

Toffalini, E., Giofrè, D., \& Cornoldi, C. (2017). Strengths and Weaknesses in the Intellectual Profile of Different Subtypes of Specific Learning Disorder: A Study on 1,049 Diagnosed Children. Clinical Psychological Science, 5(2), 402-409.

Tressoldi, P. E., Vio, C., Lorusso, M. L., Facoetti, A., \& Iozzino, R. (2003). Confronto di efficacia ed efficienza tra trattamenti per il miglioramento della lettura in soggetti dislessici. Psicologia Clinica Dello Sviluppo, (3), 481-494.

Turkeltaub, P. E., Benson, J., Hamilton, R. H., Datta, A., Bikson, M., \& Coslett, H. B. (2012). Left lateralizing transcranial direct current stimulation improves reading efficiency. Brain Stimulation, 5(3), 201-207.

Vellutino, F. R., Fletcher, J. M., Snowling, M. J., \& Scanlon, D. M. (2004). Specific reading disability (dyslexia): what have we learned in the past four decades? Journal of Child Psychology and Psychiatry, 45(1), 2-40.

Vicario, C. M., \& Nitsche, M. A. (2013). Transcranial direct current stimulation: a remediation tool for the treatment of childhood congenital dyslexia? Frontiers in Human Neuroscience, 7.

Wolf, M., Bowers, P. G., \& Biddle, K. (2000). Naming-Speed Processes, Timing, and Reading: A Conceptual Review. Journal of Learning Disabilities, 33(4), 387-407.

World Health Organization. (1992). The ICD-10 classification of mental and behavioural disorders: clinical descriptions and diagnostic guidelines (Vol. 1). World Health Organization. 
